\title{
HPV-16 E6 and E7 oncogene expression is downregulated as a result of Mdm2 knockdown
}

\author{
DANIEL DIAZ ${ }^{1}$, MAURICIO A. SALINAS SANTANDER ${ }^{2}$ and JESÚS A. MORLETT CHAVEZ ${ }^{1}$ \\ ${ }^{1}$ Department of Clinic Analysis and Molecular Diagnostic, Faculty of Chemistry, Universidad Autónoma \\ de Coahuila, Av. Venustiano Carranza/José Cárdenas, Republica Oriente, CP 25280, Saltillo, \\ Coahuila; ${ }^{2}$ Department of Biochemistry and Molecular Medicine, Faculty of Medicine, \\ Universidad Autónoma de Nuevo León, Monterrey, Nuevo León, Mexico
}

Received November 30, 2011; Accepted February 3, 2012

DOI: 10.3892/ijo.2012.1429

\begin{abstract}
The carcinogenic potential of HPV infections is based on the integration and constitutive expression of the E6 and E7 genes which inhibit the $\mathrm{p} 53$ and $\mathrm{Rb}$ tumor suppressor proteins. In normalcells, $\mathrm{Mdm} 2$ regulates $\mathrm{p} 53$ in a negative feedback loop, and although Mdm2 is apparently functional in HPV-infected cells, E6 is the protein responsible for repressing p53 replacing Mdm2 function. The role of Mdm2 in HPV-positive cells is still elusive. In this study, Mdm2 was knocked down in an HPV-positive cervical cancer cell line; as a result we found downregulation of the expression of E6 and E7 and p53 upregulation.
\end{abstract}

\section{Introduction}

The infection with human papilloma virus (HPV) has been associated with cervical cancer in $90 \%$ of cases (1); over 200 serotypes of HPV have been identified but only 23 are known to infect the human cervical tract and of these, only 13 are considered 'high risk' (2-4). HPV-16 and -18 are found in the latter category and between them are responsible for over $70 \%$ of neoplasias, HPV-16 being responsible of $41-54 \%$ of cervical carcinomas worldwide $(5,6)$.

The carcinogenic potential of HPV is attributed to the partial or total integration of the viral DNA into the host cell genome, resulting in the constitutive expression of E6 and E7 which inhibit p53 and pRb tumor suppressor activity (7), although it is known that E6 has other carcinogenic activities independent of its interaction with p53 $(8,9)$.

Basal $p 53^{w t}$ expression can be found in HPV-positive cancer cells, and although the $p 53^{\mathrm{Arg} 72}$ allele is more susceptible to E6

Correspondence to: Dr Daniel Diaz, Departamento de Análisis Clínicos y Diagnostico Molecular, Facultad de Ciencias Químicas, Universidad Autónoma de Coahuila, Av. Venustiano Carranza esquina con José Cárdenas, Republica Oriente, CP 25280, Saltillo, Coahuila, Mexico

E-mail: daniel.diazg@gmail.com

Key words: cervical cancer, E6, E7, HPV-16, Mdm2, RNAi mediated degradation than its $p 53^{\text {Pro72 }}$ counterpart (12), cervical carcinomas in which both HPV infection and mutant alleles of p53 coincide are rarely seen $(10,11)$.

It has been suggested that E6 expression levels are closely related to p53 activity (13) and that the continuous expression of the E6 and E7 genes in transformed HPV-positive cells is required to maintain their malignant state (14); therefore, p53 stabilization in these cells by inhibiting E6 or E7 expression has been attempted in the past (15).

On the other hand, Mdm2 has been identified as an E3 ligase capable of binding and exporting p53 outside the nucleus (16) where it is degraded by ubiquitin thereby inhibiting p53 tumor suppressor activities such as growth arrest or apoptosis (17-19), hence it is known as the natural repressor of p53. In transformed HPV-infected cells, however, p53-Mdm2 interaction is impaired and Mdm2 is completely replaced by E6 in the process of nuclear export and ubiquitin mediated degradation of p53 (20). The role of Mdm2 in these cells has not been entirely determined yet. It is suggested, however, that Mdm2 may associate with the HPV E2 protein enhancing the viral protein's transcriptional activity (21).

In the last decade, the use of RNA interference (RNAi) has been widely applied in diverse studies. In HPV-infected cancer cells, RNAi silencing of E6 and E7 has been done with different results $(7,15,22)$; for instance, the co-suppression by RNAi of both E6 and E7 in HPV-infected cells has been found to enhance the effect of growth arrest in these cells than otherwise obtained by silencing only one of them (23), on the other hand, the gene knockdown of E6, Mdm2 and other p53 antagonists shows a higher apoptosis rate than silencing only E6 (24).

The side effect of inhibiting E6 in HPV-transformed cells is the accumulation and stabilization of p53 and the subsequent changes in the expression of some of the p53-dependent genes, such as Bax, which is necessary for apoptosis in HPV-infected cervical cancer cell lines (25). However, by knocking down E6 in HPV-infected lung cancer cell lines, the expression level of Mdm2, which is downregulated in these cells, was restored to similar levels found in HPV-negative cells (26).

Regardless, the effect that silencing Mdm2 could have over the expression of p53, E6 and E7 and why the co-suppression of $\mathrm{Mdm} 2$ and the E6 oncogene enhances apoptosis in HPV-infected cervical cancer cells has not been fully addressed. In this study 
we found that the RNAi knockdown of Mdm2 in a cervical cancer cell line not only results in the upregulation of p53 but also in downregulation of E6 and E7 gene expression.

\section{Materials and methods}

Plasmids. Short hairpin RNA (shRNA) were expressed from vector pSilencer 1.0 (Ambion, Austin, TX). The zeocin drug resistance cassette was amplified by PCR from the pcDNA4/ TO plasmid (Invitrogen, Carlsbad, CA) using the primers 5'-AACGGGGTACCGGAACAACACTCAACCCTATCT-3' and 5'-GCGGCGGGTACCCCAGACATGATAAGATACAT TGATG-3' and cloned in the KpnI site of pSilencer 1.0. The shRNA target sequences were designed using the siRNA Design Tool from Ambion and further screened for thermodynamic stability (27). The designed shRNA DNA oligonucleotides consisted of the ApaI cloning sequence, 19 nucleotides (nt) of the target sequence followed by a 9-nt loop sequence (TTCAA GAGA), the reverse complement to the 19-nt target sequence, the stop codon for the U6 promoter (TTTTT), and the EcoRV restriction sequence. The sequence used for Mdm2 was 5'-CG CCACAAATCTGATAGTA-3'; and as an RNAi target sequence for EGFP which was also used as a control RNAi sequence we used 5'-GAAGCAGCACGACTTCTTC-3'. The complementary strands were mixed in an equimolar ratio $(10 \mu \mathrm{M})$ in annealing buffer (1X PBS pH 6.8, $2 \mathrm{mM} \mathrm{MgCl}_{2}$ ), heated at $95^{\circ} \mathrm{C} / 5 \mathrm{~min}$ and cooled at room temperature. The shRNA constructs were cloned in the $\mathrm{pSilencer}_{\mathrm{zeo}}$ ApaI/Eco RV restriction sites.

Cell lines and transfections. SiHa epithelial cell line is derived from a human cervical carcinoma and contains 1-2 copies of HPV-16 genome integrated per cell. It was grown and maintained in DMEM supplemented with $10 \%$ fetal bovine serum and $100 \mathrm{U} / \mathrm{ml}$ penicillin, $100 \mu \mathrm{g} / \mathrm{ml}$ streptomycin, $0.25 \mu \mathrm{g} / \mathrm{ml}$ amphotericin B (Invitrogen). For transfection, the cells were trypsinized and bedded in 24 -well plates, $5 \times 10^{5}$ cells per well. After $24 \mathrm{~h}$ the cells were transfected with Lipofectamine 2000 (Invitrogen) according to the manufacturer's instructions. The cells were trypsinized $48 \mathrm{~h}$ later and bedded in 12-well plates; stable selection was done adding zeocin $(100 \mu \mathrm{g} /$ $\mathrm{ml}$ ) for 15 days. The cells were genotyped by PCR using the primers: U6fwd (5'-GGTACCCGCTCTAGAACTAGTGG-3'), U6rvs (5'-AAACAAGGCTTTTCTCCAAGGG-3') and pSrvs (5'-TTACGCCAAGCGCGCAATTAAC-3') to verify construct integrity. Thermal cycle was $94^{\circ} \mathrm{C} / 30 \mathrm{sec} ; 59^{\circ} \mathrm{C} / 30 \mathrm{sec}$; $72^{\circ} \mathrm{C} / 1 \mathrm{~min}$ ( 35 cycles).

GFP assay. Cells were cultured in a 24-well plate and transfected using Lipofectamine 2000 (Invitrogen) with $300 \mathrm{ng}$ of the pEGP-N3 plasmid (Clontech, Mountain View, CA); $500 \mathrm{ng}$ RNAi control; 500 ng pS-shRNAGFP; 500 ng mock vector; and either $300 \mathrm{ng}$ of the pEGP-N3 plus $500 \mathrm{ng}$ pS-shRNAGFP; or $500 \mathrm{ng}$ pS-shRNAmdm2. At $72 \mathrm{~h}$ after transfection the cells were harvested, washed twice with $1 \mathrm{X}$ PBS, placed in a slide and analyzed in a Leica DB1000 confocal microscope. This experiment was repeated at least three times.

RNA purification. Cells were grown in $60-\mathrm{mm}$ plates, washed twice with 1 X PBS pH 7.5 and total RNA was extracted using

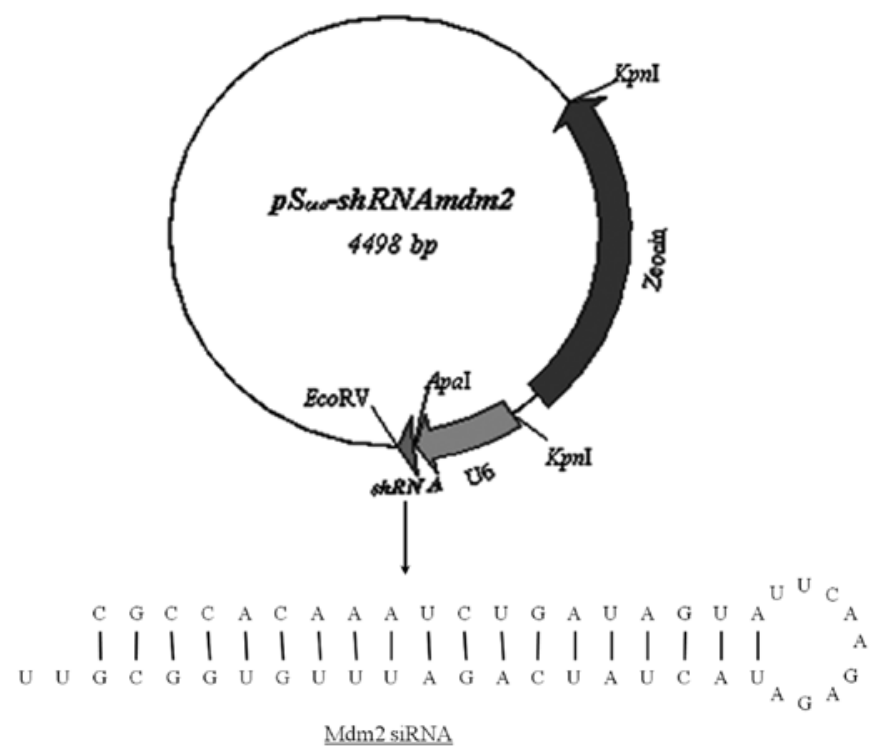

Figure 1. RNAi expression vector. The RNAi constructs for Mdm2 and GFP were annealed and cloned in the ApaI/EcoRV restriction sites of the pSilencer 1.0 plasmid. These constructs are driven by the murine U6 promoter. Also, the zeocin resistance gene was cloned in the KpnI site of the vector to obtain stably transfected cell lines.

TRIzol (Invitrogen) according to the manufacturer's instructions. RNA integrity was verified in $1 \%$ agarose (MOPS)-formaldehyde gel electrophoresis.

$R T-P C R$. Total RNA was treated with RQ1 RNase-Free DNase (Promega, Madison, WI) according to the manufacturer's instruction. Single strand cDNA was obtained from $4 \mu \mathrm{g}$ of total RNA using AMV Reverse Transcriptase (New England Biolabs, Ipswich, MA) following the manufacturer's instructions.

Semi-quantitative PCR. For semi-quantitation, $200 \mathrm{ng}$ of cDNA was amplified in multiplex reaction using Taq polymerase (GenScript, Piscataway, NJ); the following primers were used: for GAPDH, 5'-AGGTCGGAGTCAACGGATTTGGT-3' and 5'-CATGTGGGCCATGAGGTCCACCAC-3'; for Mdm2, 5'-GCGCGAAAACCCCGGATGGTGAGG-3' and 5'-CCTG TGCTCTTTCACAGAGAAGC-3'; for p53, 5'-GGTTCACTG AAGACCCAGGTCC-3' and 5'-ACCATCGCTATCTGAGCA GCGC-3'; for E6 5'-CAGGACCCACAGGAGCGACCC-3' and 5'-GGACACAGTGGCTTTTGACAG-3'; and for E7, 5'-CCCAGCTGTAATCATGCATG-3' and 5'-CCCATTAAC AGGTCTTCCAAAGTACG-3'. The thermal cycle used was: $94^{\circ} \mathrm{C} / 30 \mathrm{sec}, 59^{\circ} \mathrm{C} / 30 \mathrm{sec}, 72^{\circ} \mathrm{C} / 1 \mathrm{~min}$. Twenty-eight cycles were used for Mdm2; 29 cycles for p53; and 25 cycles for E6 and E7. Mdm2, p53, E6, and E7 DNA bands were quantified and normalized against the corresponding GAPDH bands to obtain relative expression levels.

Statistical methods. Densitometry data from DNA bands was obtained using Gel Pro Analyzer 4.0 software (Media Cybernetics), these data were analyzed using a Student's twotailed t-test. Mean average and standard deviation of at least four independent experiments is given; significance was set at $\leq 0.05$. 

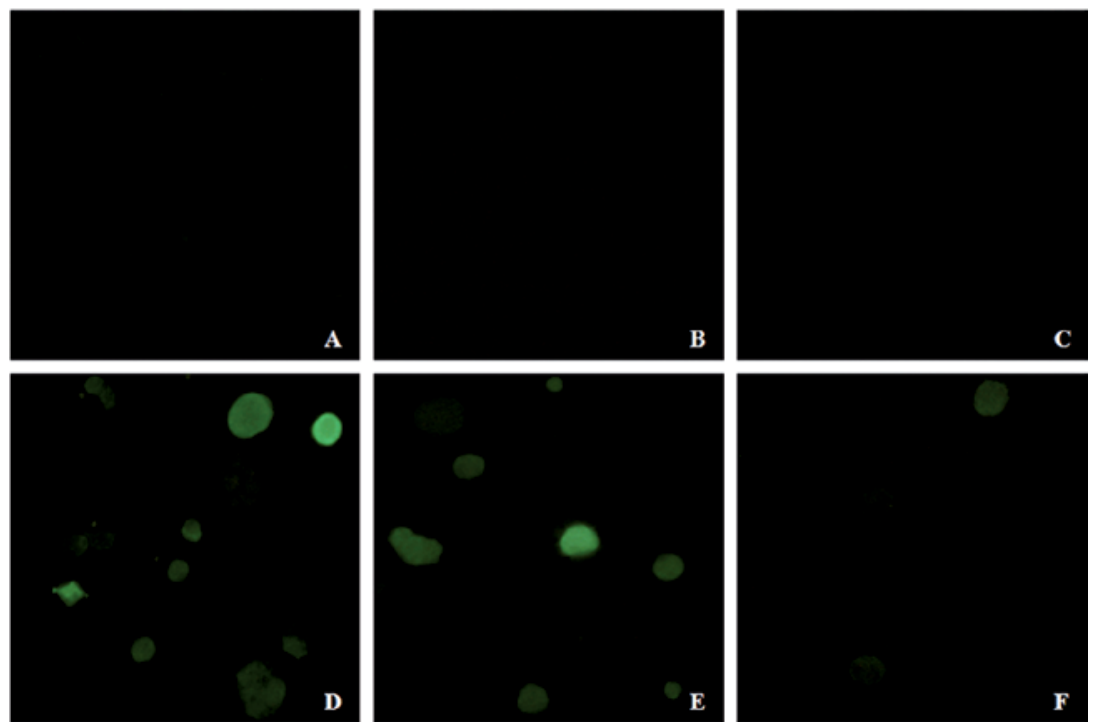

Figure 2. GFP assay. To verify the expression of the RNAi constructs in SiHa cells; the mock vector (A), RNAi-mdm2 (B), RNAi-GFP (C) and the GFP reporter were transfected alone (D); or co-transfected the reporter plus the RNAi-mdm2 (E) or the RNAi-GFP expression plasmids (F). Only when co-transfecting the RNAi-GFP vector, reduction in GFP expression can be seen $(\mathrm{F})$ as opposed when co-transfecting the RNAi-mdm2 plasmid, where no effect on GFP expression is noted (E).

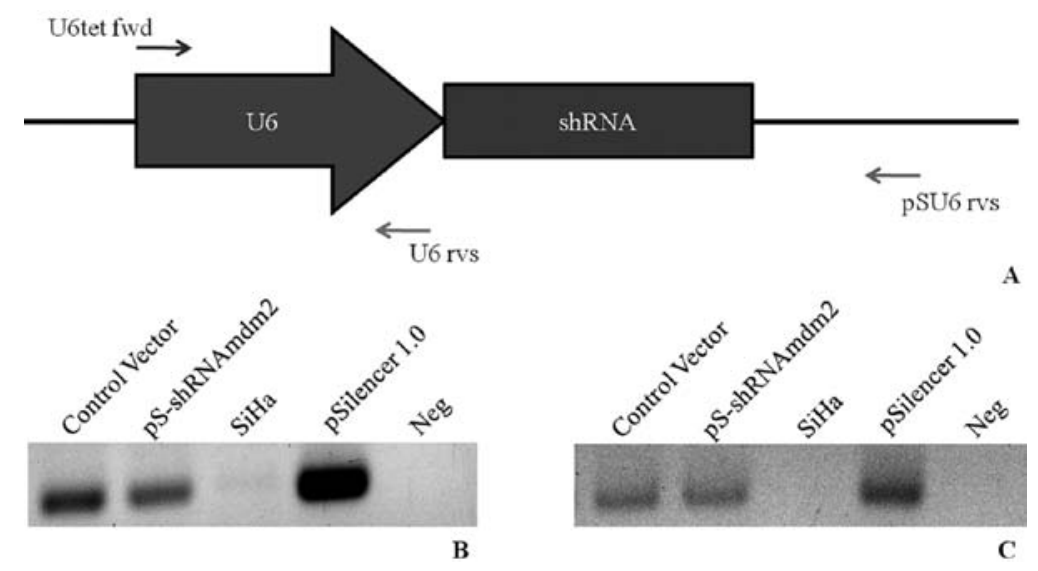

Figure 3. Genotyping of stable cell clones. The stably transfected cell clones were genotyped, U6fwd-U6rvs detects the transgenic murine U6 promoter, whereas U6fwd-pSU6rvs determines if the RNAi construct was also integrated into the host cell genome (A). PCR detection of the U6 promoter in stably transfected cell lines, control vector represents the clones transfected with the RNAi-GFP plasmid; SiHa cells were used as a negative control and the pSilencer 1.0 plasmid was used as positive control for the PCR reaction (B). To determine construct integrity in the transfected cells, the pSU6rvs primer was used, this primer is annealed downstream of the RNAi construct (C).

\section{Results}

shRNA constructs and GFP assay. The Mdm2 shRNA target sequence was selected based in the thermodynamic stability of its 3'-end, which would facilitate the entry of the anti-sense strand into the RISC complex (27), this construct also meets 5 out of 7 (28) and 4 out of 4 (29) criteria for siRNA design. This siRNA targets position 858 of the $h m d m 2$ mRNA (PubMed access: M92424). The target sequence for EGFP was designed in a similar fashion. These shRNA constructs were cloned in the pSilencer 1.0 vector, which has the murine RNApolIII promoter, U6 (Fig. 1).

To verify the functionality of the murine U6 promoter in $\mathrm{SiHa}$ cells as well as the general design of our shRNA constructs, we transfected a GFP reporter plasmid alone in SiHa cells (Fig. 2D), and co-transfected the GFP reporter plasmid with either the shRNAmdm 2 or shRNAGFP constructs.
After $72 \mathrm{~h}$ of transfection, GFP expression was efficiently suppressed when co-transfected with pS-shRNAGFP (Fig. 2F) but not when co-transfected with the pS-shRNAmdm 2 vector (Fig. 2E); showing that the RNAi constructs were expressed in SiHa cells using a U6 murine promoter and that the design of the siRNA sequences was adequate.

Stably transfected cell lines and genotyping. It was previously noted that, in our assays, transfection efficiency was very low in SiHa cells so, in order to obtain more uniform data, the zeocin resistance gene was amplified from the pcDNA4/TO plasmid and cloned in the shRNA expression vectors. After transfecting the RNAi plasmids, the SiHa cells were selected using the drug zeocin for 15 days.

The cell lines were later genotyped amplifying the murine U6 promoter by PCR (Fig. 3A). The primers U6fwd and U6rvs were used to amplify the U6 promoter in the stably transfected 


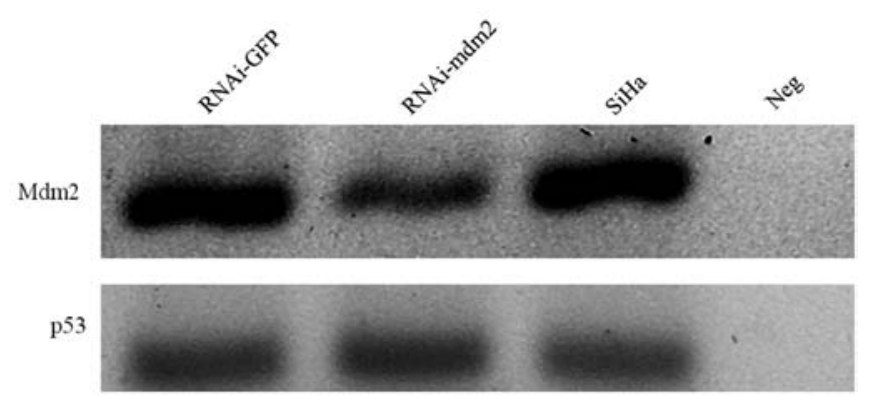

E6

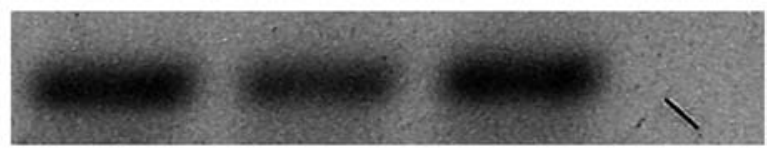

E7

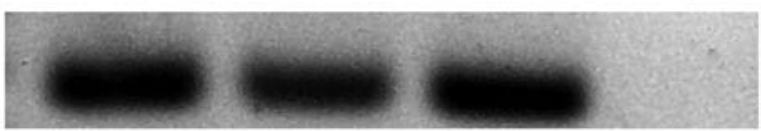

GAPDH

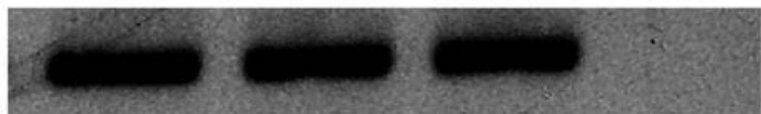

Figure 4. Semi-quantitative PCR analysis. Semi-quantitative PCR reactions were resolved in a $0.8 \%$ agarose gel, they were later analyzed by densitometry and the band intensity values obtained of each gene was normalized against GAPDH expression; these experiments were repeated at least four times.

cells (Fig. 3B); the positive clones were further screened using the U6fwd and pSU6rvs primers, the latter anneals to a plasmid sequence immediately downstream of the shRNA construct, hence it would determine if the RNAi sequence was also integrated into the host cell genome (Fig. 3C).
Semi-quantitative PCR analysis. After obtaining the stable $\mathrm{SiHa}$ cells, two clones of each transfected construct were chosen at random. Total RNA was obtained from these clones and from untransfected $\mathrm{SiHa}$ cells which were used as an untreated control. The cDNA was obtained in a reverse transcription assay and a multiplex amplification curve was done to establish the cycle number in which each gene was to be analyzed (data not shown).

The shRNAmdm2, control shRNAGFP cell clones and SiHa cells were cultured in 60-mm plates, and harvested after $72 \mathrm{~h}$, total RNA was extracted, and cDNA synthesis was performed; $200 \mathrm{ng}$ of this cDNA was later used to amplify mdm2, p53, E6 and E7 making a semi-quantitative analysis by testing band intensity against GAPDH (Fig. 4).

The analysis of Mdm2 showed that mRNA level had diminished $65 \%$ when compared with control shRNAGFP clones $(\mathrm{p} \leq 0.05)$ and against untreated SiHa cells ( $\mathrm{p} \leq 0.05)$ (Fig. 5), showing effective Mdm2 knockdown.

It has been previously reported that $\mathrm{Mdm} 2$ silencing does not, or only in a negligible manner, result in the activation of p53 $(24,30)$. In agreement with previous reports, our stably transfected shRNAmdm 2 cell lines did not present any of the characteristics of p53 activity, such as growth arrest or apoptosis.

However, the effect that mdm 2 knockdown alone could have on p53 expression in a cervical cancer cell line has not yet been reported to the best of our knowledge. So, with this in mind we analyzed the expression level of p53 in these cells; we found that, although p53 activation is not apparent in the cells that have Mdm2 knockdown, the mRNA level of p53 is upregulated, showing a $56 \%$ increase when compared with the untreated SiHa cells or with the control shRNAGFP cells $(\mathrm{p} \leq 0.05)$ (Fig. 5). Although p53 expression was increased, it is

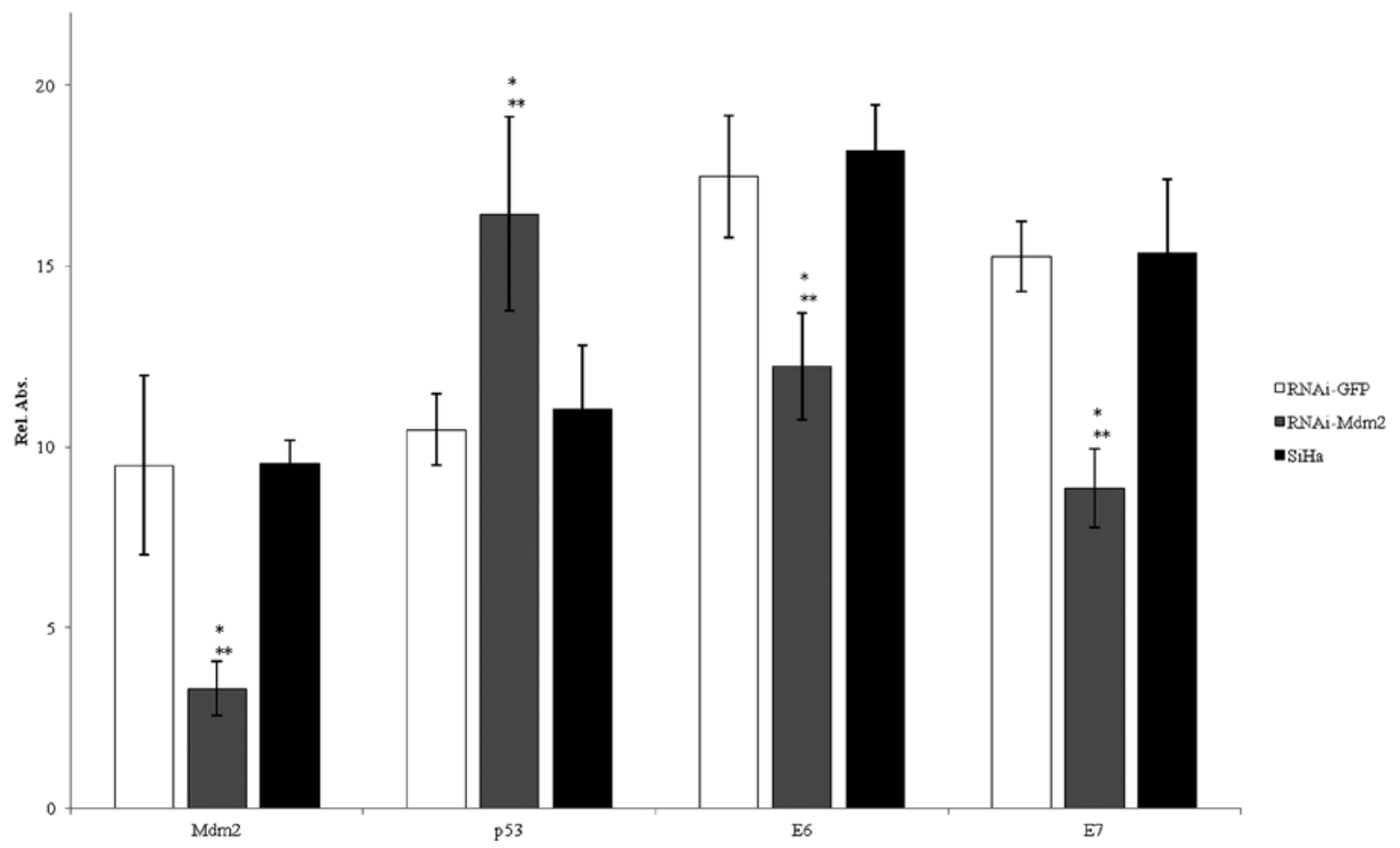

Figure 5. Statistical analysis. Densitometric analysis of DNA bands helped us determine relative expression of the analyzed genes; the average mean from at least four different experiments is represented as columns in this graph; the horizontal axis represents which gene is being amplified by PCR and the vertical axis represents the relative absorbance values obtained by densitometry analysis. "Treatment vs. RNAi control p $\leq 0.05$. ${ }^{* *}$ Treatment vs. cell line control p $\leq 0.05$. 
likely that its transcription activity could not be achieved since E6 expression levels are in close relation to those of p53 activity (13).

Trying to determine how E6 and E7 mRNA levels behaved in response to p53 upregulation by Mdm2 knockdown, we analyzed the treated and untreated cells. Unexpectedly, we found that E6 mRNA level had decreased 30\% when compared against the untreated and control shRNAGFP cells $(\mathrm{p} \leq 0.05)$; moreover, the level of E7 mRNA expression was also decreased by $42 \%$ when compared with the untreated and control cells (p $\leq 0.05$ ) (Fig. 5).

These results show that by silencing Mdm2 using RNAi in an HPV-infected cervical cancer cell line, the expression level of p53, E6 and E7 are modified. The obtained results were somewhat unexpected and though p53 mRNA expression is upregulated and E6 and E7 mRNA levels are decreased, no growth arrest or apoptosis effect was apparent.

\section{Discussion}

The data obtained in this study showed that the inhibition of Mdm2 using RNAi, results in expression changes of p53, E6 and E7 in an HPV-infected cervical cancer cell line. The tumor suppressor activity of p53 in an HPV-infected cell is impaired by E6; moreover, E6 completely replaces Mdm2 in the nuclear export and ubiquitin mediated degradation of p53 in these cells (20); on the other hand, E7 can inhibit pRb tumor suppressing activities by preventing its binding to the E2F transcription factors and promoting its degradation $(31,32)$.

The oncogenic potential of E6 and E7 is dependent upon their constitutive expression, which is required to preserve the malignant state of the infected cell (14); therefore, to overcome this effect has been tried by stabilizing p53 through a number of different strategies, among them, RNAi $(7,15,22)$. However, the results obtained in these works differ in their conclusions; other approaches have determined that the inhibition of E6 is not sufficient in itself to promote p53 activation and that it is also necessary to induce p53 response by additional means, such as genotoxic treatment (33) or p53 antagonists co-suppression (24).

On the other hand, it has been shown that the stabilization and activation of $\mathrm{p} 53$ is negligible by inhibiting Mdm2 expression in an HPV-infected cervical cancer cell line $(24,30)$, therefore, although Mdm 2 knockdown is enough to upregulate the expression of p53, and downregulate E6 and E7, it is not sufficient to elicit p53 tumor suppressing activities, since no senescence, growth arrest or apoptotic effect was observed.

While the co-suppression of E6 and Mdm2 has been found to potentially increase the apoptotic effect that would be otherwise obtained by silencing only E6 (24); the fact that the knockdown of Mdm2 alone downregulates E6 and E7 in an HPV-infected cell line is somewhat baffling and hints at a not yet fully understood relation between these proteins that may be influenced by previously described Mdm2/E2 transcription activity (21).

In regards to $\mathrm{E} 2$, its open reading frame codes a $\sim 42-\mathrm{kDa}$ protein that regulates viral transcription through its binding to DNA palindromic sequences present in several copies in the long controlling region (LCR) of all papillomaviruses (reviewed in ref. 34) and it is also involved in the viral replication cycle by collaborating with E1 and cellular replication factors (35). E2 binds to multiple sites in the regulatory region (URR) control- ling the expression of early promoters in a positive (low E2 concentration) or negative (high E2 concentration) way (36). However, the E2 transcriptional activity is not completely clear and it seems that its transcription role depends on the HPV serotype.

The E2 protein is the transcriptional activator element for E6 and E7 expression in HPV-16 and, according to Gammoh et al (21), the E2 association with Mdm2 allows it to reach its full transcriptional potential; from this point of view, it makes sense that in knocking down Mdm2, the expression levels of E6 and E7 could be downregulated.

We consider that further research in this regard could improve the opportunity to fully understand the relationship between Mdm2 and HPV oncogene regulation and provide enlightenment to alternative therapy strategies against HPV-infected cervical cancer cells.

\section{Acknowledgements}

SiHa cell line was kindly provided by Dr J.M. Alcocer. Special thanks to Dr Tomoo Iwakuma who supplied the pSilencer 1.0 vector and advice; to Dr Pablo Zapata who provided the pEGFP-N3 reporter plasmid. This work was supported by the Consejo Nacional de Ciencia y Tecnología (CONACyT), grant no. 49196-M.

\section{References}

1. Bosch FX, Manos MM, Munoz N, Sherman M, Jansen AM, Peto J, et al: Prevalence of human papillomavirus in cervical cancer: a worldwide perspective. J Natl Cancer Inst 87: 796-802, 1995.

2. Münger K and Howley PM: Human papillomavirus immortalization and transformation functions. Virus Res 89: 213-228, 2002.

3. Greenblatt RJ: Human papillomaviruses: diseases, diagnosis, and a possible vaccine. Clin Microbiol Newslett 27: 139-145, 2005.

4. Sinal SH and Woods CR: Human papillomavirus infections of the genital and respiratory tracts in young children. Semin Pediatr Infect Dis 16: 306-316, 2005.

5. Noel JC, Lespagnard L, Fayt I, Verhest A and Dargent JL: Evidence of human papilloma virus infection but lack of Epstein-Barr virus in lymphoepithelioma-like carcinoma of uterine cervix: report of two cases and review of the literature. Hum Pathol 32: 135-138, 2001.

6. Baseman JG and Koutsky LA: The epidemiology of human papillomavirus infections. J Clin Virol 32: 16-24, 2005.

7. Goodwin EC and DiMaio D: Repression of human papillomavirus oncogenes in HeLa cervical carcinoma cells causes the orderly reactivation of dormant tumor suppressor pathways. Proc Nat Acad Sci USA 97: 12513-12518, 2000.

8. Kubbutat MH and Vousden KH: New HPV E6 binding proteins: dangerous liaisons? Trends Microbiol 6: 173-175, 1998.

9. Rapp L and Chen JJ: The papillomavirus E6 proteins. Biochim Biophys Acta 1378: F1-F19, 1998.

10. Park DJ, Wilczynski SP, Paquette RL, Miller CW and Koeffler HP: p53 mutations in HPV-negative cervical carcinoma. Oncogene 9: 205-210, 1994.

11. Denk C, Butz K, Schneider A, Dürst M and Hoppe-Seyler F: p53 mutations are rare events in recurrent cervical cancer. J Mol Med 79: 283-288, 2001.

12. Zehbe I, Voglino G, Wilander E, Delius H, Marongiu A, Edler L, et al: p53 codon 72 polymorphism and various human papillomavirus 16 E6 genotypes are risk factors for cervical cancer development. Cancer Res 61: 608-611, 2001.

13. Butz K, Whitaker N, Denk C, Ullmann A, Geisen C and HoppeSeyler F: Induction of the p53-target gene GADD45 in HPV-positive cancer cells. Oncogene 18: 2381-2386, 1999.

14. Von Knebel Doeberitz M, Rittmuller C, zur Hausen H and Durst M: Inhibition of tumorigenicity of cervical cancer cells in nude mice by HPV E6-E7 anti-sense RNA. Int J Cancer 51: 831-834, 1992. 
15. Hietanen S, Lain S, Krausz E, Blattner C and Lane DP: Activation of p53 in cervical carcinoma cells by small molecules. Proc Natl Acad Sci USA 97: 8501-8506, 2000.

16. Momand J,Zambetti GP, Olson DC, George D and Levine AJ: The mdm-2 oncogene product forms a complex with the p53 protein and inhibits p53-mediated transactivation. Cell 69: 1237-1245, 1992.

17. Finlay CA: The $m d m-2$ oncogene can overcome wild-type p53 suppression of transformed cell growth. Mol Cell Biol 13: 301-306, 1993

18. Chen J, Wu X, Lin J and Levin AJ: mdm-2 inhibits the G1 arrest and apoptosis functions of the $\mathrm{p} 53$ tumor suppressor protein. Mol Cell Biol 16: 2445-2452, 1996.

19. Haupt Y, Barak Y and Oren M: Cell type-specific inhibition of p53-mediated apoptosis by mdm2. EMBO J 15: 1596-1606, 1996

20. Hengstermann A, Linares LK, Ciechanover A, Whitaker NJ and Scheffner M: Complete switch from Mdm2 to human papillomavirus E6-mediated degradation of p53 in cervical cancer cells. Proc Natl Acad Sci USA 98: 1218-1223, 2001.

21. Gammoh N, Gardiol D, Massimi P and Banks L: The Mdm2 ubiquitin ligase enhances transcriptional activity of human papillomavirus E2. J Virol 83: 1538-1543, 2009.

22. Hall AH and Alexander KA: RNA interference of human papillomavirus type 18 E6 and E7 induces senescence in HeLa cells. J Virol 77: 6066-6069, 2003.

23. Gu W, Putral L, Hengst K, Minto K, Saunders NA, Leggatt G, et al: Inhibition of cervical cancer cell growth in vitro and in vivo with lentiviral-vector delivered short hairpin RNA targeting human papillomavirus E6 and E7 oncogenes. Cancer Gene Ther 13: 1023-1032, 2006.

24. Koivusalo R, Mialon A,Pitkänen H, Westermarck J and Hietanen S: Activation of $\mathrm{p} 53$ in cervical cancer cells by human papillomavirus E6 RNA interference is transient, but can be sustained by inhibiting endogenous nuclear export-dependent $\mathrm{p} 53$ antagonists. Cancer Res 66: 11817-11824, 2006

25. Vogt M, Butz K, Dymalla S, Semzow J and Hoppe-Seyler F: Inhibition of Bax activity is crucial for the antiapoptotic function of the human papillomavirus E6 oncoprotein. Oncogene 25: 4009-4015, 2006.

26. Cheng YW, Wu MF, Wang J, Yeh KT, Goan YG, Chiou HL, et al: Human papillomavirus 16/18 E6 oncoprotein is expressed in lung cancer and related with p53 inactivation. Cancer Res 67: 10686-10693, 2007.
27. Khvorova A, Reynolds A and Jayasena SD: Functional siRNAs and miRNAs exhibit strand bias. Cell 115: 209-216, 2003.

28. Reynolds A, Leake D, Boese Q, Scaringe S, Marshall WS and Khvorova A: Rational siRNA design for RNA interference. Nat Biotechnol 22: 326-330, 2004.

29. Ui-Tei K, Naito Y, Takahashi F, Haraguchi T, Ohki-Hamazaki H, Juni A, et al: Guidelines for the selection of highly effective siRNA sequences for mammalian and chick RNA interference. Nucleic Acids Res 32: 936-948, 2004.

30. Traidej M, Chen L, Yu D, Agrawal S and Chen J: The roles of E6-AP and MDM2 in p53 regulation in human papillomaviruspositive cervical cancer cells. Antisense Nucleic Acid Drug Dev 10: 17-27, 2000.

31. Boyer SN, Wazer DE and Band V: E7 protein of human papilloma virus-16 induces degradation of retinoblastoma protein through the ubiquitin-proteasome pathway. Cancer Res 56: 4620-4624, 1996.

32. Gonzalez SL, Stremlau M, He X, Basile JR and Münger K: Degradation of the retinoblastoma tumor suppressor by the human papillomavirus type $16 \mathrm{E} 7$ oncoprotein is important for functional inactivation and is separable from proteasomal degradation of E7. J Virol 75: 7583-7591, 2001.

33. Koivusalo R, Krausz E, Helenius H and Hietanen S: Chemotherapy compounds in cervical cancer cells primed by reconstitution of p53 function after short interfering RNA-mediated degradation of human papillomavirus 18 E6 mRNA: opposite effect of siRNA in combination with different drugs. Mol Pharmacol 68: 372-382, 2005.

34. Ham J, Dostatni N, Gauthier JM and Yaniv M: The papillomavirus E2 protein: a factor with many talents. Trends Biochem Sci 16: 440-444, 1991.

35. Del Vecchio AM, Romanczuk H, Howley PM and Baker CM: Transient replication of human papillomavirus DNAs. J Virol 66: 5949-5958, 1992.

36. Demeret C, Desaintes C, Yaniv M and Thierry F: Different mechanisms contribute to the E2-mediated transcriptional repression of human papillomavirus type 18 viral oncogenes. J Virol 71: 9343-9349, 1997. 